\title{
Electromagnetic modelling of a human eye exposed to conductive keratoplasty
}

\author{
A. Peratta \\ Wessex Institute of Technology, UK
}

\begin{abstract}
This paper presents 3D numerical solutions of the current density, absorbed energy and initial impedance of the human eye exposed to conductive keratoplasty (CK) scenarios obtained with the boundary element method. CK is a non-ablative surgical technique for the treatment of mild to moderate hyperopia (far-sightedness). In a CK session a thin electrode penetrates the cornea delivering pulsed radiofrequency energy at $350 \mathrm{kHz}$ to the surrounding tissue. The electromagnetic (EM) energy is dissipated into heat in the tissue surrounding the tip yielding thermally localised shrinkage and tightening of the collagen llamelae. When applied in a controlled way, this relatively new technique allows eye surgeons to correct the shape of the cornea and to treat common types of eye disease such as far-sightedness or astigmatism. This work examines in detail the induced currents that appear in the rest of the eye while delivering the EM signal. Quantitative estimations of the EM energy absorbed in each tissue per unitary voltage apart from the cornea is presented, as well as the impedance of the electrodes at initial time.
\end{abstract}

Keywords: human eye, conductive keratoplasty, boundary element method, electric field.

\section{Introduction}

There is a great deal of interest in understanding the electrical properties of the human eye in the low frequency (LF) range, and how it behaves as a complex imperfect conductive body. Studies consider the eye as either a passive element exposed to an external field, or as an active one which produces a complicated pattern of electrical signals in response to light. The former is usually tackled in order to understand the vulnerability of the different tissues when exposed to potentially harmful levels of electric fields at different frequencies, orders of magnitude and 
exposure times, while the latter is usually studied to further our current understanding on how vision works, i.e. to develop machines capable of simulating the electric response of the photosensitive tissues.

On the other hand, the development of safe and effective thermal techniques to alter the topography of the cornea for correcting vision defects has been challenging ophthalmologists for more than hundred years.

Localised high temperatures can induce shrinkage in the corneal collagen. In addition, an oscillating electric field can be absorbed by biological tissues and used to produce heat in a controlled way. This is the principle of the recently successful technique based called Conductive Keratoplasty (CK) [1-4]. Hence, the accurate prediction of the LF electric fields and current densities in the different tissues is fundamental. This requires solving the macroscopic Maxwell equations in an assembly of biological tissues with non-homogeneous conductivity and permittivity. For this purpose, numerical modelling is one of the most useful approaches.

In time harmonic EM fields, Maxwell equations can be decoupled when the characteristic size of the model (2-3 cm for the human eye) is much smaller than the characteristic vacuum wavelength $\left(\lambda_{0} \sim 860 \mathrm{~m}\right.$ at frequency $\left.\nu=350 \mathrm{kHz}\right)$ and the displacement currents can be neglected in comparison to the resistive ones. Therefore, the high voltage LF approach is applicable and the numerical problem summarises into solving the non-homogeneous Laplace equation.

On the other hand, the Boundary Element Method (BEM) $[5,6]$ is a well established numerical technique for accurate solutions even in complex geometries. The beauty of BEM is that it takes into account the fundamental solution of the leading partial differential operator, and that the discretisation can be done only in the boundary of the problem. The BEM has been successfully applied for solving problems of biological tissues, other than the human eye, exposed to low and high frequency electromagnetic (EM) fields [7-10]. The objective of this paper is to present the BEM solution of LF EM fields in a 3D model of the human eye.

\section{Low frequency EM modelling with boundary elements}

When considering biological tissue exposure to high voltage and low currents the most influential field is the electric one. Assuming both conductivity $\sigma$ and permittivity $\varepsilon$ to be constant within a finite region of interest (sub-domain) it is derived from Maxwell's equations that the electric scalar potential $\varphi$ obeys the non-homogeneous Laplace equation: $\nabla \cdot[(\sigma+i \omega \varepsilon) \nabla \varphi]=0$, where $e^{i \omega t}$-time dependency convention has been used, being $\omega=2 \pi \nu$ the angular frequency of the incident field, $\varepsilon$ the permittivity, and $i^{2}:=-1$. The corresponding integral formulation for the potential $\varphi\left(\mathbf{x}_{\mathbf{s}}\right)$ at point $\mathbf{x}_{\mathbf{s}} \in \Omega$ satisfies the following boundary integral equation $[5,6]$ :

$$
c_{s} \varphi\left(\mathbf{x}_{s}\right)+\int_{\Gamma} E_{n}^{*}\left(\mathbf{x}, \mathbf{x}_{s}\right) \varphi(\mathbf{x}) d \Gamma-\int_{\Gamma} \varphi^{*}\left(\mathbf{x}, \mathbf{x}_{s}\right) E_{n}(\mathbf{x}) d \Gamma=0,
$$

where $\Omega$ is the integration domain with boundary $\Gamma=\partial(\Omega)$ of outward unit normal $\hat{\mathbf{n}}, \varphi^{*}$ is the Green's function of Laplace equation: $\nabla^{2} \varphi^{*}+\delta\left(\mathbf{x}_{s}, \mathbf{x}\right)=0$, 
and $E_{n}^{*}$ its normal derivative in $\hat{\mathbf{n}}$ direction. Also, proper boundary conditions are applied to $\Gamma=\partial(\Omega)$, i.e. Dirichlet, Neumann or Robin type. In 3D space $\varphi^{*}$ and $E_{n}^{*}$ become: $\varphi^{*}=1 /(4 \pi r)$ and $E_{n}^{*}=\mathbf{r} \cdot \hat{\mathbf{n}} /\left(4 \pi r^{3}\right)$, respectively; where $\mathbf{r}=\mathbf{x}-\mathbf{x}_{s}, r=|\mathbf{r}|$ is the distance between the field $(\mathbf{x} \in \Gamma)$ and source $\left(\mathbf{x}_{s} \in \Omega\right)$ points, and $c_{s}$ is a constant dependent on the Cauchy principal value integration of the singularity at the source point. After discretisation with flat linear triangular elements, eq. (1) becomes:

$$
c_{s} \varphi_{s}+\sum_{e=1}^{N_{e}} \sum_{a=1}^{3} h^{(e, a)} \varphi_{e, a}-\sum_{e=1}^{N_{e}} g^{(e)} E_{n, e}=0
$$

where $\varphi_{e, a}$ and $E_{n, e}$ denote potential and normal electric field, respectively at $a$ th continuous node in $e$-th element, and $q^{(e)}$ and $h^{(e, a)}$ are the usual BEM single and double layer integrals [6]. The assembly of (1) leads to a linear system of equations $\mathbf{A} \mathbf{x}=\mathbf{b}$, which solution provides the missing $\varphi$ and $E_{n}$ values at the boundary. At LF, biological tissues behave as good conductors with conductivity values of the order of $0.5 \mathrm{~S} / \mathrm{m}$, and electric permittivity $10^{-10} \mathrm{~F} / \mathrm{m}$, i.e. $\varepsilon_{r} \sim 100$; and the air represents a nearly perfect dielectric. Since air has negligible conductivity in comparison with tissues, and the permittivity of most biological tissues is few orders of magnitude greater than $\varepsilon_{0}$ [11]. Therefore, appropriate matching conditions between air $(0)$ and tissue $(1,2)$ and different tissues, can be written as:

$$
\left[\sigma \frac{\partial \varphi_{I}}{\partial n}\right]_{1,2}=\left[\omega \varepsilon \frac{\partial \varphi_{R}}{\partial n}\right]_{0} \quad ;\left[\sigma \frac{\partial \varphi_{I}}{\partial n}\right]_{1}=\left[\sigma \frac{\partial \varphi_{I}}{\partial n}\right]_{2},
$$

respectively; where $\mathbf{j} \cdot \hat{\mathbf{n}}$ is preserved, $\mathbf{j}=\sigma \nabla \varphi$ and is current density.

\section{Conceptual model}

Conductive Keratoplasty (CK) [1-4] is a non-ablative surgical technique for the treatment of mild to moderate hyperopia. In a CK session a thin electrode ( $\sim 90 \mu \mathrm{m}$ wide, $450 \mu \mathrm{m}$ long) penetrates the cornea and delivers pulsed radio-frequency energy at $350 \mathrm{kHz}$ to the stroma, which forms majority of the thickness of the cornea structure $[12,13]$. The EM energy is dissipated into heat in the tissue surrounding the tip yielding thermally localised shrinkage and tightening of the collagen llamelae in a controlled way. When heat is applied as a ring of spots around the mid peripheral cornea, a constricting band is formed which induces steepening in its central part, and consequent decrease of cornea's radius of curvature. In this way, the cornea is reshaped and its focusing properties can be permanently corrected. The BEM approach has been used in order to solve the EM part of the problem (equi-potential surfaces of voltage and induced current densities in the different tissues) of the CK treatment in a three dimensional model of the human eye, allowing a better understanding of the distribution of heat loss in the eye.

Fig. 1 shows the top and lateral views of the model. The horizontal and vertical axes in the lateral view indicate distance in $\mathrm{mm}$. The origin of the model 


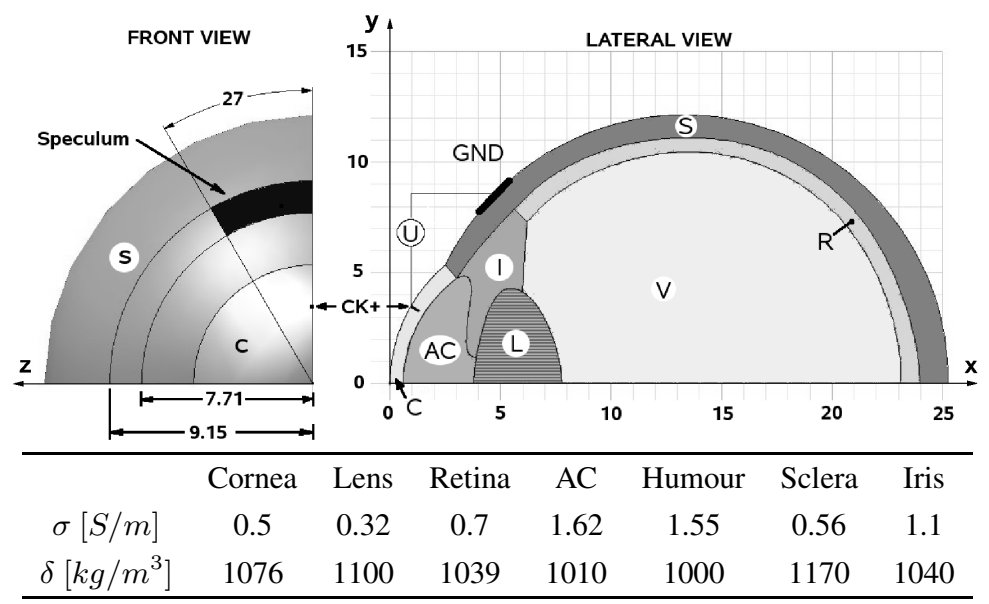

Figure 1: CK model of the human eye (dimensions are in $\mathrm{mm}$.) $\mathrm{CK}$ indicates the location of the active CK electrode. Conductivity $(\sigma)$ and mass density $(\delta)$ of the tissues involved were taken from refs [14-16].

$\{0,0,0\}$, in the $\{x, y, z\}$ system of coordinates, is indicated in Fig. 1 for further reference. The $45 \mu \mathrm{m}$ (width) $\times 950 \mu \mathrm{m}$ (long) electrode, indicated as $\mathrm{CK}+$ in the picture, is located at a radial distance of $3.5 \mathrm{~mm}$ from the centre of the cornea (i.e. $y=3.5 \mathrm{~mm}$ ). The speculum used to retract the eyelids during the $\mathrm{CK}$ session acts as the return (dispersive) electrode. In this work, it has been assumed that the contact area between the sclera and speculum is approximately $2 \mathrm{~mm}$ in width by $4 \mathrm{~mm}$ long. The voltage difference applied between speculum and CK electrode is $1 \mathrm{~V}$. Then, because of the linearity assumed in this model, all results of current density, heat loss and voltage must be scaled up accordingly. The geometrical data has been obtained from references $[17,18]$. The conductivities of the different tissues were adopted from existing literature [11,14-16]. The lens plays a key role in the model since its conductivity is considerable lower than the surrounding tissues. Motivated by Arrhenius type rate reactions, Pearce et al [12] assumed an exponential dependence of the form: $\sigma(T, w)=\sigma_{0} w \exp \left[0.015\left(T-T_{0}\right)\right]$, while in this work $\sigma$ has been considered constant, for the sake of simplicity, and the distribution produced will reflect the real field at initial time only, i.e. during the first few milliseconds after the voltage is established.

The geometrical information of the eye is entered as a wireframe composed of arcs and curves, patched with NURBSurfaces. The voids in between define a subdomain. The model is composed of 7 sub-domains: cornea, lens, retina, anterior chamber $(\mathrm{AC})$ vitreous humour, sclera and iris. Then, the boundaries are discretised with flat triangles by means of a Finite Elements Method - type mesh generator $[19,20]$. A 3D view of the meshed model can be observed in Fig. 2. To reduce as much as possible the number of degrees of freedom, the model considers a quarter of the eye, including 2396 nodes and 5262 elements yielding a linear system of 


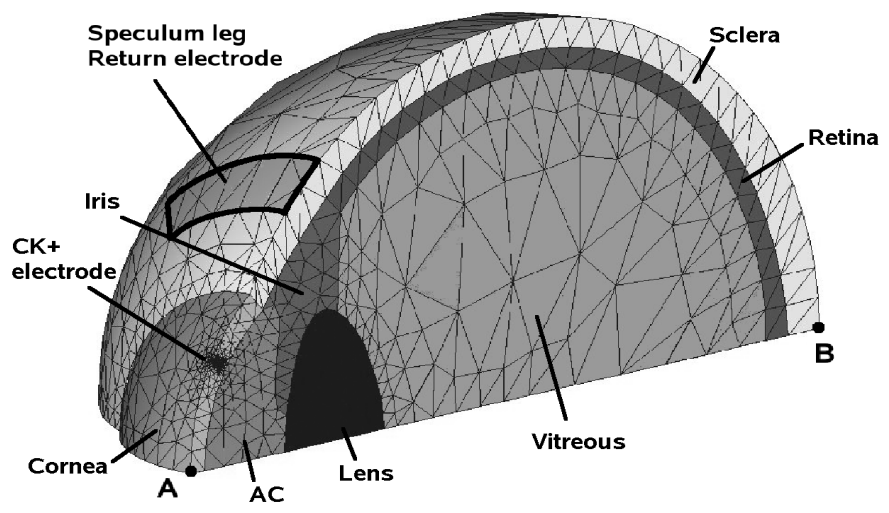

Figure 2: 3D quarter model of the human eye. 2639 triangular staggered elements and 1180 continuous nodes, 7 subdomains.

7718 equations and 4502 unknowns. The system took $11 \mathrm{~min}$ to solve in a Pentium 4 PC - 3GHz CPU - 512 MBytes RAM, with the Lapack direct solver from [21].

The flat boundaries and the outer skin of the sclera and cornea have adiabatictype boundary conditions, this is to consider the $90^{\circ}$ rotation symmetry around central axis ( $\overline{A B}$ in Fig. 2). A non-symmetric model should provide the opportunity to introduce asymmetric features in the model, such as the central retinal artery, optical nerve, and hyaloid canal [17].

This model is a convenient simplification of the eye. Relevant regions such as the cilliary part of the retina, the tear layer and the choroid layer between sclera and retina have been neglected. The choroid has high density of blood vessels and it is expected to behave as a highly conductive thin layer. The cilliary part is considered as included in the iris in this present model in view of the similar conductivity, and the tear layer should play a key role when considering convection in the thermal part of the CK problem.

\section{Results}

Figure 3 shows the potential distribution obtained with the BEM approach. The top left figure shows the surface distribution in the cornea and sclera, as well as the tissue surrounding the leg of the speculum in contact with the eye from the front view. The top right figure shows equi-potential lines in a slice passing through the centre of the eye from a lateral view. The bottom right figure shows the equipotential lines in a sagital slice through the centre of the eye from the bottom view. The non-uniformity of the voltage distribution obtained is a result from the different conductivities of the model. The epithelium is a one-cell layer thick in charge of pumping water from the cornea for keeping it clear and slightly dry, as a requirement for optical transparency. It is important that this layer is not exposed to 


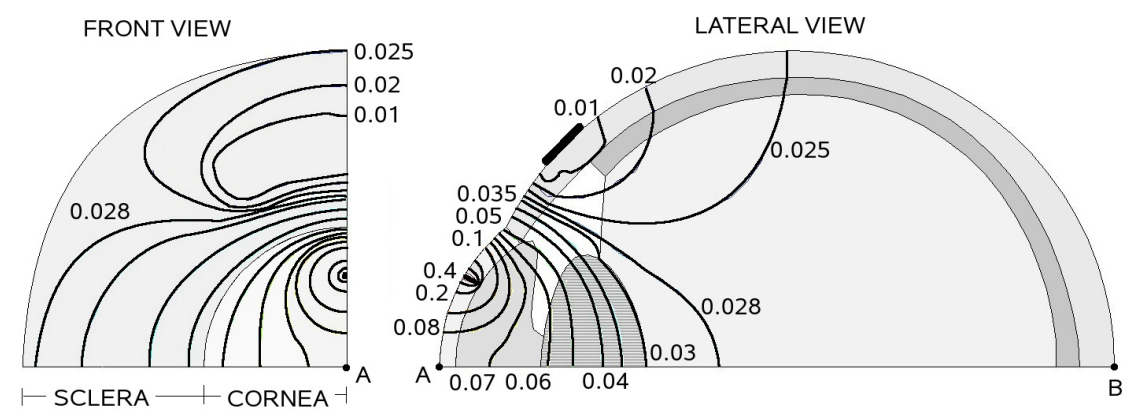

$X, Y, Z$ in $\mathrm{mm}$

Point A: $(0,0,0)$

Point B: $(25.3,0,0)$

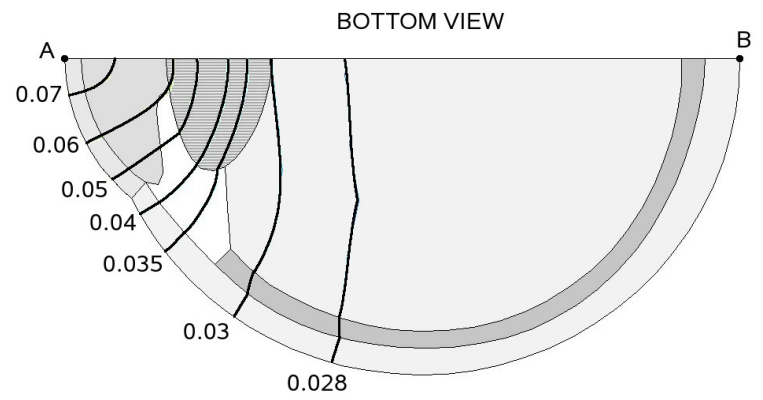

Figure 3: Iso-surfaces distribution of potential $\varphi$, for a unitary voltage applied between speculum leg and CK electrode.

high current densities. Numerical results indicate that the current density has a non negligible component tangential to this layer. The maximum absolute value found in this layer is $|\mathbf{j}|=0.7 \times 10^{3} \mathrm{~A} / \mathrm{m}^{2}$ per applied volt, and is found underneath the CK electrode. The maximum value of current density found in the lens is $\sim$ $5.7 \mathrm{~A} / \mathrm{m}^{2}$ per unitary voltage.

The results obtained from the simulation allow to compute the effective impedance $(Z=1740 \Omega)$ given by: $Z=\Delta V\left[2 \sum_{i=1}^{n}(\mathbf{j} \cdot \hat{\mathbf{n}})_{i} A_{i}\right]^{-1}$, where $A_{i}$ $(i=1, \ldots, n)$ is the area of the $\mathrm{i}$-th element in the electrode, $n=250$ is the number of elements in the electrode, $\Delta V=1$ is the applied voltage between speculum leg and CK electrode, and the factor 2 in the denominator is because that the model involved half space. The total initial impedance obtained in this work is $Z_{0}=1740 \Omega$, which compares relatively well with the findings from Berjano et al [13] $1561 \Omega$ and the experimental values measured by Choi et al [22] in porcine eyes $1600 \Omega, 1775 \Omega, 2158 \Omega$.

The modulus of the current density in the cornea decays approx. exponentially with the distance $\rho$ to the CK electrode according to: $f(\rho)=A \exp [-\lambda(\rho-0.2)]$, where $A \sim 0.4 \mathrm{~A} / \mathrm{m}^{2}$ and $\lambda \sim 2 \mathrm{~mm}^{-1}$ were obtained with a least squares approach from the numerical results. The power absorbed $I$ at any point in the cornea decreases with the radial distance $(\rho)$ to the CK electrode according to: $\frac{I}{I_{0}}=\exp [-2 \lambda \rho]$, with a characteristic length $L=1 /(2 \lambda)=0.25 \mathrm{~mm}$. 
Table 1: Energy absorbed in each tissue at initial time, per unitary applied voltage.

\begin{tabular}{llllll}
\cline { 1 - 2 } & $V\left[\mathrm{~mm}^{3}\right]$ & $\langle p\rangle[W / \mathrm{kg}]$ & AC & 131.6 & 0.610 \\
\cline { 1 - 2 } Humour & 4150.0 & 0.00094 & Retina & 864.7 & 0.002156 \\
Lens & 142.3 & 0.027 & Sclera & 1664.0 & 0.0158 \\
Iris & 298.0 & 0.06577 & Cornea & 64.0 & 14.6 \\
\cline { 6 - 7 }
\end{tabular}

The specific power absorbed in the tissue, considered as heat loss per unit time and volume, is given by $p=\mathbf{j} \cdot \mathbf{E}$. Then, the total energy rate absorbed in the tissue within sub-domain $\Omega_{s}$ is the integral $P=\int_{\Omega_{s}} p d \Omega_{s}$. One of the beauties of $\mathrm{BEM}$, is that volumetric quantities such as $P$ can be accurately evaluated with the information on the boundary $\Gamma_{s}=\partial\left(\Omega_{s}\right)$, according to Green's first identity:

$$
P=\int_{\Omega_{s}} \mathbf{j} \cdot \mathbf{E} d \Omega_{s}=\int_{\Gamma_{s}} \varphi \mathbf{j} \cdot \hat{\mathbf{n}} d \Gamma_{s}
$$

The volume $V$ of each sub-domain can be computed as: $V=1 / 3 \int_{\Gamma_{s}} \mathbf{x} \cdot \hat{\mathbf{n}} d \Gamma_{s}$. Then, the specific mean EM power absorbed in each tissue $\langle p\rangle$ can be estimated according to:

$$
\langle p\rangle=\frac{P}{V} \approx 3 \frac{\sum_{i=1}^{n} \varphi_{i}(\mathbf{j} \cdot \hat{\mathbf{n}})_{i} A_{i}}{\sum_{i=1}^{n}(\mathbf{x} \cdot \hat{\mathbf{n}})_{i} A_{i}}
$$

The results obtained numerically for the absorbed energy in the different tissues at initial time are summarised in Table 1. It is worth mentioning that as time evolves, the physical properties including conductivity, water content, and thermal diffusion coefficient of the different tissues vary. Hence, the power distribution shown in Table. 1 is only valid at initial time. Nevertheless, this is enough to have a quantitative estimation of the energy absorbed in each tissue. Figure 4 shows the distribution of absorbed power (heat loss) in the front of the eye due to the CK electrode with unitary voltage, i.e. $\Delta V=1 V$. A logarithmic scale has been adopted for $\langle p\rangle$, in view of the wide range of values observed. Figure 5 shows the heat loss profile along $x$ direction at three different observation lines. The first one located at $y=0 \mathrm{~mm}$ (longitudinal axis of the eye), the second at $y=3.3 \mathrm{~mm}$, corresponding to the same level as the CK electrode (see Fig. 4), and the third named "Retina" is a $10.2 \mathrm{~mm}$ radius arc with centre at $\{x, y, z\}=\{13.3,0.656,0\}$ fully contained in the retina. The results for the observation line $y=3.3 \mathrm{~mm}$ show that the heat loss near the CK electrode in the cornea is of the order $O\left(10^{4}\right) \mathrm{W} / \mathrm{kg}$, roughly about 4 orders of magnitude greater than in the AC. The maximum absorbed power at the central line $(y=0 \mathrm{~mm})$ found in the front of the lens, at approximately $x=4 \mathrm{~mm}$, is $\langle p\rangle=0.04 \mathrm{~W} / \mathrm{kg}$. For $x>x_{0}$, the absorbed power decreases nearly with the exponential law: $\langle p\rangle=A_{0} \exp \left[-\alpha\left(x-x_{0}\right)\right] ; \quad x>x_{0}$, where $x_{0}=5 \mathrm{~mm}, A_{0}=0.43 \mathrm{Wkg}^{-1}$ and $\alpha=0.63 \mathrm{~mm}^{-1}$, were obtained by exponential fit with least squares from the computed data (see Fig. 5). In addition, the 

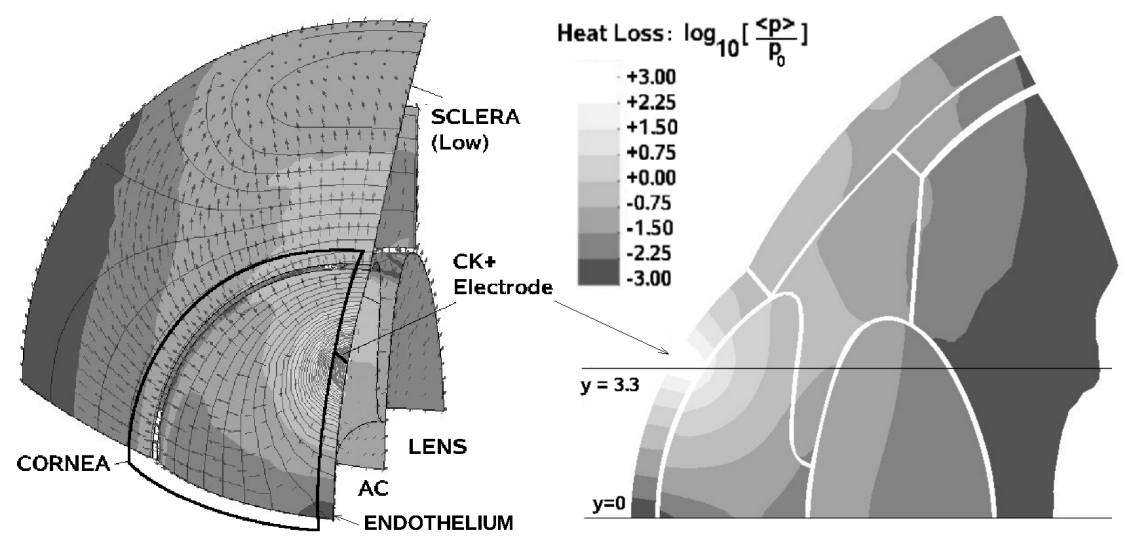

Figure 4: Density plot of heat loss distribution, in combination with equi-potential lines (continuous lines) and density current (vectors), for unitary voltage applied between speculum leg and $\mathrm{CK}+$ electrode. The numbers in the bar scale indicate $\log _{10}\left[\frac{\langle p\rangle}{p_{0}}\right]$, where $p_{0}=1 \mathrm{~W} / \mathrm{kg}$.

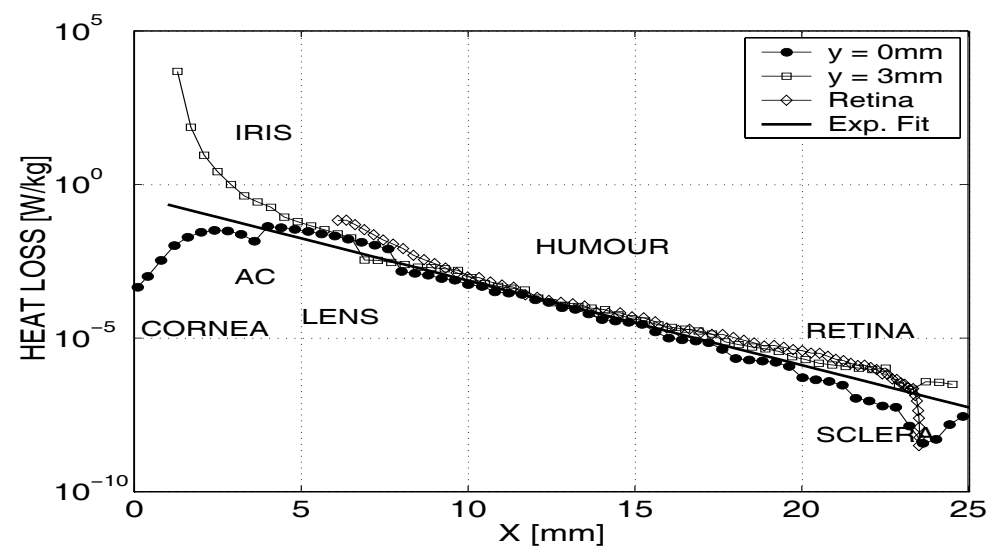

Figure 5: Heat loss vs. $x$ coordinate at three observation lines: $y=0$ (eye's longitudinal axis); $y=3 \mathrm{~mm}$ (near CK electrode tip); and "Retina",(10.2 $\mathrm{mm}$ radius arc fully contained in the retina. The continuous line represents the exponential fit in the region $21 \mathrm{~mm}>x>5 \mathrm{~mm}$.

maximum exposure in the retina, found in the closest region to the CK electrode, is $\langle p\rangle=0.065 \mathrm{~W} / \mathrm{kg}$, then it decreases nearly exponentially with $x$. The macula is exposed to absorption rates of the order $O\left(10^{-6}\right) \mathrm{W} / \mathrm{kg}$ per unitary applied volt. A value that should be easily dissipated by blood perfusion. 


\section{Conclusions}

The BEM has been successfully applied in order to calculate heat loss, induced currents, voltage and electric fields in a three dimensional model of the human eye exposed to $\mathrm{CK}$ treatment. The method is able to compute the distribution of current density and absorbed power within the eye including far and near fields. These results help to estimate the relative exposure level of other tissues, like the lens, humour vitreous, sclera, iris anterior chamber and retina in addition to the cornea. The maximum EM power is absorbed in the cornea and in the anterior chamber. The order of magnitude of the maximum heat loss in the retina is $O\left(10^{-2}\right) \mathrm{W} / \mathrm{kg}$ per unitary voltage applied between the CK electrode and the speculum leg. This maximum was found in the peripheral region of the retina near the CK electrode. The heat loss in the macula is of the order $O\left(10^{-6} \mathrm{~W} / \mathrm{kg}\right.$ per applied volt. This amount should be effortlessly dissipated by biological thermal regulatory mechanisms, i.e. blood perfusion.

Local computational models truncated at a distance of $\sim 10 \mathrm{~mm}$ from the $\mathrm{CK}$ electrode should be acceptable for predicting near field heat loss and current densities in the cornea.

\section{References}

[1] G. A. Mendez and A. Mendez Noble. Conductive keratoplasty for the correction of hyperopia, chapter 17, pages 163-171. Williams and Wilkens, Baltimore, 1997.

[2] I. Pallikaris, T. Naoumidi, and N. Astyrakakis. Long-term results of conductive keratoplasty for low to moderate hyperopia. J Cataract Refractive Surg, 31, August 2005.

[3] D. Lin and E. Manche. Two-year results of conductive keratoplasty for the correction of low to moderate hyperopia. J Cataract Refract Surg, 29:23392350, 2003.

[4] M. McDonald et al. Conductive Keratoplasty for the Correction of Low to Moderate Hyperopia: US Clinical Trial 1-Year Results on 355 Eyes. Am. Academy of Ophthalmology, pages 1978-1989, 2002.

[5] C. Brebbia, J. Telles, and L. Wrobel. Boundary Elements Techniques. Splinger-Verlag, Berlin, Heidelberg, New York and Tokio, 1984.

[6] C. Brebbia and J.Dominguez. Boundary Elements, an Introductory Course. Computational Mechanics Publications. McGraw-Hill, New York, Colorado, San Francisco, Mexico, Toronto, $2^{\text {nd }}$ edition, 1992.

[7] N. Kovac D.Poljak, Choy Yoong Tham. The assesment of human exposure to low frequency and high frequency electromagnetic fields using boundary element analysis. Engineering Analysis with Boundary Elements, 27:9991007, 2003.

[8] M. C. Gonzalez, A. Peratta, and D. Poljak. Boundary Element Modeling of the Realistic Human Body Exposed to Extremely-Low-Frequency (ELF) 
Electric Fields: Computational and Geometrical Aspects. IEEE Transactions on Electromagnetic Compatibility, 49(1):153-162, 2007.

[9] A. Peratta, C.Gonzalez, and D. Poljak. Geometrical aspects of 3d human body exposed to extremely low frequency electromagnetic fields. In 14th Int. Conf on Sofware, Telecomm and Comp. Networks, Split CROATIA, 2006.

[10] A. Peratta, C. Gonzalez, and D. Poljak. Current density induced in the human body due to power distributions lines using the boundary element method. J.of Comm. Soft. and Syst., 3(1), 2007.

[11] R.W.Lau S.Gabriel and C.Gabriel. The dielectric properties of biological tissues: II. measurements in the frequency range $10 \mathrm{hz}$ to $20 \mathrm{ghz}$. Phys. Med. Biol., 41:2251-2269, 1996.

[12] J. Pearce, D. Panescu, and S Thomsen. Simulation of diopter changes in radio frequency conductive keratoplasty. In M. Ursino, C. Brebbia, and E. Magosso, editors, Modelling in Med. and Biol. VI, volume 2 of Advances in Bioengng, pages 469-477, Southampton, UK, 2005. Wessex Institute of Technology, WIT Press.

[13] E. Berjano, J. Alió, and J. Saiz. Modeling for radio-frequency conductive keratoplasty: implications for the maximum temperature reached in the cornea. Phys. Measurement, 26:157-172, January 2005.

[14] G. Lindemblatt and J. Silny. A model of the electrical volume conductor in the region of the eye in the ELF range. Phys. Med. Biol., 46:3051-3059, October 2001.

[15] A. Barchanski, M. Clemens, H. De Gersem, and T. Weiland. Efficient calculation of current densities in the human body induced by arbitrarily shaped, low-frequency magnetic field sources. Journal of Computational Physics, 214:81-95, November 2006.

[16] A. Hirata, S. Matsuyama, and T. Shiozawa. Temperature rises in the human eye exposed to EM waves in the frequency range 0.6-6 GHz. IEEE Transactions on Electromagnetic Compatibility, 42(4):386-393, November 2000.

[17] D. Smerdon. Anatomy of the eye and orbit. Current Anaesthesia and Critical Care, 11:286-292, 2000.

[18] E. Hermans, M. Dubbelman, G. van der Heijde, and R. Heethaar. Estimating the external force acting on the human eye lens during accommodation by finite element modelling. Vision Research, 2006. Available online at www.sciencedirect.com.

[19] GID resources. Website. http://gid.cimne.upc.es.

[20] CIMNE, International Center for Numerical Methods in Engineering, Barcelona, Spain. GID, The personal pre/postprocessor Manual.

[21] E. Anderson, Z. Bai, C. Bischof, S. Blackford, J. Demmel, J. Dongarra, J. Du Croz, A. Greenbaum, S. Hammarling, A. McKenney, and D. Sorensen. LAPACK Users' Guide. Society for Industrial and Applied Mathematics, Philadelphia, PA, third edition, 1999.

[22] B. Choi, A. J. Welch, and J.A. Pearce. Dynamic impedance measurements during radio-frequency heating of cornea. IEEE Trans. Biomed. Engng., 49(6):1610, 2002. 